\title{
On measures of classicality/quantumness in quasiprobability representations of finite-dimensional quantum systems
}

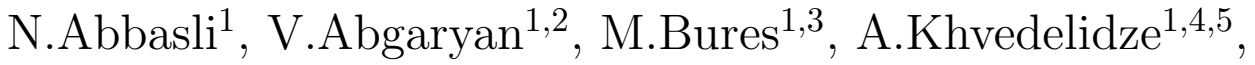 \\ I.Rogojin $^{1}$ and A.Torosyan ${ }^{1}$ \\ ${ }^{1}$ Laboratory of Information Technologies, JINR, Dubna, Russia \\ ${ }^{2}$ A.I. Alikhanyan National Science Laboratory (YerPHI), Yerevan, Armenia \\ ${ }^{3}$ Institute of Experimental and Applied Physics, CTU, Prague, Czech Republic \\ ${ }^{4}$ A Razmadze Mathematical Institute, TSU, Tbilisi, Georgia \\ ${ }^{5}$ Institute of Quantum Physics and Engineering Technologies, GTU, Tbilisi, Georgia
}

\begin{abstract}
In the present report we discuss measures of classicality/quantumness of states of finite-dimensional quantum systems, which are based on a deviation of quasiprobability distributions from true statistical distributions. Particularly, the dependence of the global indicator of classicality on the assigned geometry of a quantum state space is analysed for a whole family of Wigner quasiprobability representations. General considerations are exemplified by constructing the global indicator of classicality/quantumness for the Hilbert-Schmidt, Bures and BogoliubovKubo-Mori ensembles of qubits and qutrits.
\end{abstract}

\section{Background and motivation}

A centenary history of development of quantum theory shows a persistent request for a genuine unification of basic quantum-mechanical principles with concepts of classical statistical physics. The primary difficulties on this way are due to a fundamental ban originating from the Heisenberg canonical commutation relations, $[q, p]=\imath \hbar$, between the phase-space variables $(q, p)$. The non-vanishing Plank constant $\hbar$ impedes the existence of a function $W_{\varrho}(p, q)$, playing the role of a proper joint probability distribution of coordinates $q$ and momenta $p$ associated with a given quantum state $\varrho$. In the early years of development of quantum theory, rejection of a complete statistical 
description allowed Weyl and Wigner to formulate a phase-space representation of quantum mechanics with a quasiprobability function $W_{\varrho}(p, q)$, such that the corresponding marginals are true probability distributions of the canonically conjugate coordinates $q$ and $p$ [1, 2]. However, in contrast to proper distributions the function $W_{\varrho}(p, q)$ is not everywhere non-negative for all quantum states and thus it can only be interpreted as a quasiprobability distribution function 11 Today, despite this drawback, a description of quantum systems using the technique of quasiprobability distribution became an important source of our understanding of quantum phenomena (see e.g. [3, 4, 5] and references therein). Furthermore, perceiving the inevitability of the existence of negative values in quasiprobability representation of states as a reflection of the real "quantumness" of physical systems, studies move to a practical context, where the negativity of states is taken as a basis for building corresponding measures of nonclassicality (e.g., [6] and references therein). However, elaborating this idea we are faced with a serious complexity. Indeed, introducing an admissible indicator of classicality/quantumness, the following general requirements should be taken into account:

(I) independence of an indicator from a representation of quantum states;

(II) independence of an indicator from a quasiprobability representation of states.

While satisfying the first requirement is a relatively simple issue, it is enough to assume that an indicator is a function of a state unitary invariants, the second task is a highly nontrivial one. There exist infinitely many quasiprobability distributions and a quantum state can be negative in one representation and positive in another. In [7] it was argued that the positivity in one representation is neither a necessary nor a sufficient condition for classical description, nor the negativity of a specific representation is sufficient for nonclassicality. Considering any one of these quasiprobability representations we are not able to determine absolute criteria for the classicality/quantumness. Ideally, in order to quantify a state classicality/quantumness we need to determine characteristics which are unique for a complete family of such representations.

In the present report we will discuss both issues, (I) and (II), constructing a classicality/quantumness measure for a family of the Wigner quasiprobability representation of finite-dimensional quantum systems. We will follow approach [9, 10, 11, to the construction of the Wigner quasiprobability distributions $W_{\varrho}^{(\boldsymbol{\nu})}\left(\Omega_{N}\right)$ of an $N$-level quantum system via the dual pairing,

$$
W_{\varrho}^{(\boldsymbol{\nu})}\left(\Omega_{N}\right)=\operatorname{tr}\left[\varrho \Delta\left(\Omega_{N} \mid \boldsymbol{\nu}\right)\right],
$$

\footnotetext{
${ }^{1}$ Certainly, for some states there exists such a true statistical distribution. For example, according to the Hudson's theorem [8], a Gaussian wave function is the only pure state corresponding to a positive Wigner function.
} 
of the density matrix $\varrho \in \mathfrak{P}_{N}$ from the quantum state space $\mathfrak{P}_{N}$ :

$$
\mathfrak{P}_{N}=\left\{X \in M_{N}(\mathbb{C}) \mid X=X^{\dagger}, \quad X \geq 0, \quad \operatorname{tr}(X)=1\right\}
$$

and the Stratonovich-Weyl (SW) kernel $\Delta\left(\Omega_{N} \mid \boldsymbol{\nu}\right) \in \mathfrak{P}_{N}^{*}$ from the dual space $\mathfrak{P}_{N}^{*}$ :

$$
\mathfrak{P}_{N}^{*}=\left\{X \in M_{N}(\mathbb{C}) \mid X=X^{\dagger}, \quad \operatorname{tr}(X)=1, \quad \operatorname{tr}\left(X^{2}\right)=N\right\}
$$

Analysing algebraic equations (3), one can conclude that,

a) The phase-space $\Omega_{N}$ can be identified as a complex flag manifold, $\Omega_{N} \rightarrow \mathbb{F}_{d_{1}, d_{2}, \ldots, d_{s}}^{N}=$ $U(N) / H$, where $\left(d_{1}, d_{2}, \ldots, d_{s}\right)$ is a sequence of positive integers with sum $N$, such that $k_{1}=d_{1}$ and $k_{i+1}=d_{i+1}-d_{i}$ with $d_{s+1}=N$. The corresponding SW kernel has the isotropy group $H=U\left(k_{1}\right) \times U\left(k_{2}\right) \times U\left(k_{s+1}\right)$;

b) The isotropy group $H$ of SW kernel provides the existence of a family of Wigner distributions. The corresponding moduli space $\mathcal{P}_{N}$ represents a spherical polyhedron on $(N-2)$-dimensional sphere $\mathbb{S}_{N-2}(1)$ of radius one. Further in the text, the $s$-dimensional moduli parameter $\boldsymbol{\nu}=\left(\nu_{1}, \nu_{2}, \ldots, \nu_{s}\right), s \leq N-2$ will be used to enumerate the Wigner distributions (see details in [10],[11]).

The representation independent characteristic of the classicality can be constructed by averaging over the moduli space $\mathcal{P}_{N}(\boldsymbol{\nu})$ :

$$
\langle Q\rangle=\frac{1}{\operatorname{Vol}\left(\mathcal{P}_{N}\right)} \int_{\mathcal{P}_{N}} \mathrm{~d} \mathcal{P}_{N}(\boldsymbol{\nu}) \mathcal{Q}_{N}[\mathrm{~g} \mid \boldsymbol{\nu}]
$$

of the global indicator of classicality/quantumness $\mathcal{Q}_{N}$ defined as the relative volume ratio of the subspace $\mathcal{O}\left[\mathfrak{P}_{N}^{(+)}\right]$of the orbit space $\mathcal{O}\left[\mathfrak{P}_{N}\right]=\mathfrak{P}_{N} / S U(N)$ of the state space $\mathfrak{P}_{N}$, where the Wigner function is non-negative [12]:

$$
\mathcal{Q}_{N}[\mathrm{~g} \mid \boldsymbol{\nu}]=\frac{\int \cdots \int_{\mathcal{O}\left[\mathfrak{P}_{N}^{(+)}\right]} \mathrm{dP}_{N}(\mathrm{~g} \mid \boldsymbol{r})}{\int \cdots \int_{\mathcal{O}_{\left[\mathfrak{P}_{N}\right]}} \mathrm{dP}_{N}(\mathrm{~g} \mid \boldsymbol{r})}
$$

The total orbit space $\mathcal{O}\left[\mathfrak{P}_{N}\right]$ can be realised as the ordered $(N-1)$-simplex in the space of eigenvalues $\boldsymbol{r}^{\downarrow}=\left\{r_{1}, r_{2}, \ldots, r_{N}\right\}$ of a density matrix $\varrho$ :

$$
\mathcal{C}^{(N-1)}=\left\{\boldsymbol{r} \in \mathbb{R}^{N} \mid \sum_{i=1}^{N} r_{i}=1, \quad 1 \geq r_{1} \geq r_{2} \geq \cdots \geq r_{N-1} \geq r_{N} \geq 0\right\}
$$


while according to [12] the subspace $\mathcal{O}\left[\mathfrak{P}_{N}^{(+)}\right]$represents a dual cone of $\mathcal{O}\left[\mathfrak{P}_{N}\right]$ :

$$
\mathcal{O}\left[\mathfrak{P}_{N}^{(+)}\right]=\left\{\boldsymbol{\pi} \in \operatorname{spec}\left(\Delta\left(\Omega_{N}\right)\right) \mid\left(\boldsymbol{r}^{\downarrow}, \boldsymbol{\pi}^{\uparrow}\right) \geq 0, \quad \forall \boldsymbol{r} \in \mathcal{O}\left[\mathfrak{P}_{N}\right]\right\}
$$

with the cone defined via the dual pairing $\left(\boldsymbol{r}^{\downarrow}, \boldsymbol{\pi}^{\uparrow}\right)=r_{1} \pi_{N}+r_{2} \pi_{N-1}+\cdots+r_{N} \pi_{1}$, of $\boldsymbol{r}$ and the $N$-tuple $\boldsymbol{\pi}$ of increasing eigenvalues of SW kernel $\Delta\left(\Omega_{N} \mid \boldsymbol{\nu}\right)$.

The suggested measure of classicality (4) fulfils both conditions, (I) and (II). By the averaging procedure in (4) we fulfil requirement (II) and since the global indicator of classicality/nonclassicality is defined on the orbit space of a quantum system and therefore provides an unitary invariant measure, the requirement (I) is satisfied as well. However, the indicator (4) depends on metrical characteristics of the moduli space $\mathcal{P}_{N}$ and the orbit space $\mathcal{O}\left[\mathfrak{P}_{N}\right]$. Since the moduli space is represented by an $(N-2)$-dimensional spherical polyhedron, we suppose that the corresponding measure corresponds to uniform distribution on $\mathbb{S}_{N-1}(1)$, while the measure on the orbit space, $\mathrm{dP}_{N}(\mathrm{~g} \mid \boldsymbol{r})=\sqrt{\operatorname{det}\|\mathrm{g}\|} \mathrm{d} r_{1} \wedge \mathrm{d} r_{2} \wedge \cdots \wedge \mathrm{d} r_{N}$, is induced from the Riemannian metric $\mathrm{g}$ on the state space $\mathfrak{P}_{N}$. In the remaining part of the report we will discuss the dependence of the suggested indicator of classicality on the metric of a quantum state space. From a wide variety of special Riemannian metrics commonly used in the Quantum Statistics and Information Theory, we will analyse the Hilbert-Schmidt metric and two monotone metrics [13, 14]), the Bures and the Bogoliubov-Kubo-Mori metrics. Detailed discussion of the indicator of classicality for qubit $(N=2)$ and qutrit $(N=3)$ will be given.

\section{Riemannian geometry of state space $\mathfrak{P}_{N}$ and clas- sicality/quantumness indicator $\mathcal{Q}_{N}$}

In this section the results of our studies of the dependence of the indicator (5) on the metric of a quantum state space will be presented. We will consider a basic, HilbertSchmidt (HS) metric and two representatives of the family of the so-called monotone metrics, the Bures (B) and Bogoliubov-Kubo-Mori (BKM) metrics.

- The Hilbert-Schmidt metric • For an $N$-dimensional quantum system the infinitesimal version of the Hilbert-Schmidt distance is given by the expression:

$$
\mathrm{g}_{\mathrm{HS}}=4 \operatorname{tr}(\mathrm{d} \varrho \otimes \mathrm{d} \varrho) .
$$

For further computational aims it is convenient to rewrite (8) in terms of SVD of a density matrix, $\varrho=U D U^{\dagger}$, where $U \in S U(N)$, and $D=\operatorname{diag}\left\|r_{1}, r_{2}, \ldots, r_{N}\right\|$ with descending order of eigenvalues from the $(N-1)$-simplex (6). Here we assume that the spectrum of $\varrho$ is generic and thus the arbitrariness of $U$ is given by the isotropy group represented by the torus $T$ of $S U(N)$. In terms of SVD coordinates, the volume form factorizes into the measure $\omega_{\mathrm{SU}(\mathrm{N}) / \mathrm{T}}$ on the coset $S U(N) / T$ induced from the Haar 
measure on the $U(N)$ group manifold and the "radial part" factor which depends on the spectrum of a state only. The latter represents the Hilbert-Schmidt measure $\mathrm{dP}\left(\mathrm{g}_{\mathrm{HS}}\right)$ on the orbit space (6),

$$
\mathrm{dP}_{N}\left(\mathrm{~g}_{\mathrm{HS}} \mid \boldsymbol{r}\right)=c_{\mathrm{HS}} \delta\left(\sum_{i=1}^{N} r_{i}-1\right) \prod_{i<j}^{N}\left(r_{i}-r_{j}\right)^{2} \mathrm{~d} r_{1} \wedge \mathrm{d} r_{2} \wedge \cdots \wedge \mathrm{d} r_{N},
$$

where $c_{\mathrm{HS}}$ is a normalization constant.

- The Bures and Bogoliubov-Kubo-Mori metrics • Using the SVD decomposition of elements of $\mathfrak{P}_{N}$, the stochastically monotone metrics can be written in the following form:

$$
\mathrm{g}_{f}=\frac{1}{4} \sum_{i=1}^{N} \frac{\mathrm{d} r_{i} \otimes \mathrm{d} r_{i}}{r_{i}}+\frac{1}{2} \sum_{i<j}^{N} c_{f}\left(r_{i}, r_{j}\right)\left(r_{i}-r_{j}\right)^{2}\left(U^{\dagger} \mathrm{d} U\right)_{i j} \otimes\left(U^{\dagger} \mathrm{d} U\right)_{i j},
$$

where $c(x, y)$ is the so-called Morozova-Chentsov function; $c_{f}(x, y)=\frac{1}{y f(x / y)}$ is given by the operator monotone function $f(t)$. For the Bures and BKM metrics these functions are $f_{\mathrm{BW}}(t)=(1+t) / 2$ and $f_{\mathrm{BKM}}(t)=(t-1) / \ln t$, respectively. Having these representations, we are in a position to compare the indicators $\mathcal{Q}$ for the simplest, two- and three-level systems endowed with the above described metrics.

\subsection{Qubit}

From (3) it follows that the spectrum of SW kernel of a 2-level system is unique, spec $\left(\Delta_{2}\right)=\{(1+\sqrt{3}) / 2,(1-\sqrt{3}) / 2\}$. Its dual pairing with a 2-level density matrix $\rho=\frac{1}{2}\left[\mathbb{I}_{2}+(\boldsymbol{\xi}, \boldsymbol{\sigma})\right]$, characterized by the Bloch vector $\boldsymbol{\xi} \in \mathbb{R}^{3}$, gives the Wigner quasiprobability distribution of the qubit defined on a 2-sphere:

$$
W_{\varrho}(\boldsymbol{n})=\frac{1}{2}+\frac{\sqrt{3}}{2}(\boldsymbol{\xi}, \boldsymbol{n}), \quad \boldsymbol{n} \in \mathbb{S}^{2} .
$$

All mixed states belong to the Bloch ball, $(\boldsymbol{\xi}, \boldsymbol{\xi}) \leq 1$, while the positivity cone (7) represents qubit states inside the following ball: $(\boldsymbol{\xi}, \boldsymbol{\xi})<1 / 3$.

- The Hilbert-Schmidt metric • Taking into account the positivity domain (7) and using the expression $(9)$ for $N=2$, the indicator $\mathcal{Q}_{2}$ of the Hilbert-Schmidt qubit reduces to the ratio of two simple integrals,

$$
\mathcal{Q}_{2}\left[\mathrm{~g}_{\mathrm{HS}}\right]=\frac{\int_{0}^{\frac{1}{\sqrt{3}}} r^{2} d r}{\int_{0}^{1} r^{2} d r}=\frac{1}{3 \sqrt{3}} \approx 0.19245
$$




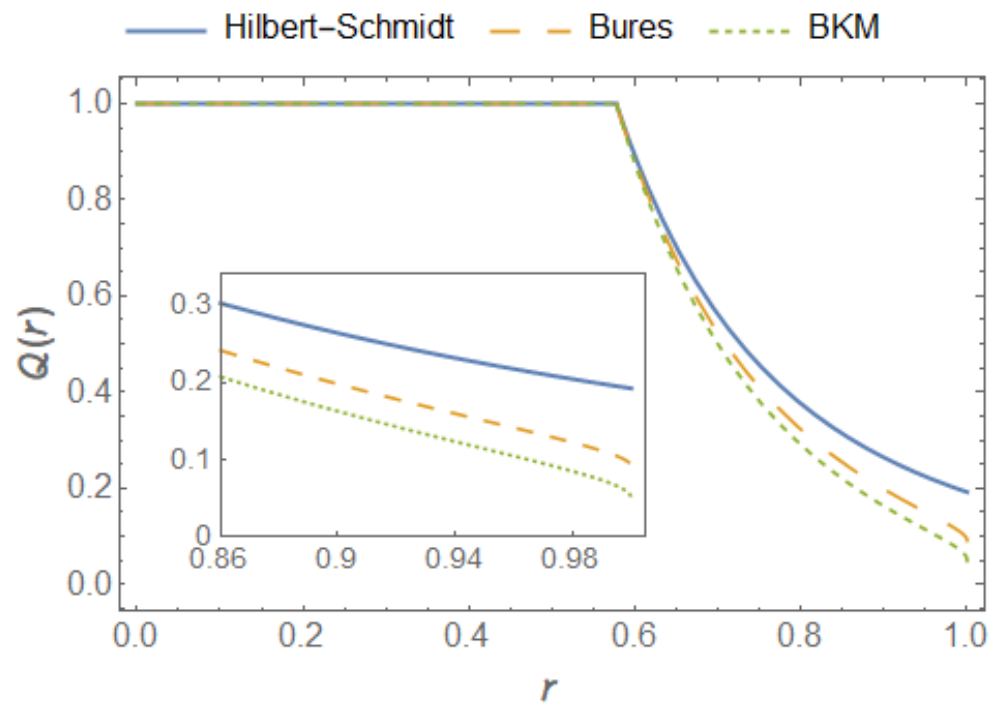

Figure 1: A qubit probability $Q(r)$, calculated for the Hilbert-Schmidt, Bures and the Bogoliubov-KuboMori metrics.

- Bures and BKM metric • Similar calculation for the Bures and BKM ensemble of qubits give,

$$
\begin{aligned}
\mathcal{Q}_{2}\left[\mathrm{~g}_{\mathrm{B}}\right] & =\frac{\operatorname{Vol}_{\mathrm{B}}\left(\frac{1}{\sqrt{3}}\right)}{\operatorname{Vol}_{\mathrm{B}}(1)}=\frac{2}{\pi}\left[\arcsin \frac{1}{\sqrt{3}}-\frac{\sqrt{2}}{3}\right] \approx 0.09172, \\
\mathcal{Q}_{2}\left[\mathrm{~g}_{\text {ВКм }}\right] & =\frac{\operatorname{Vol}_{\text {ВКм }}\left(\frac{1}{\sqrt{3}}\right)}{\operatorname{Vol}_{\text {ВКм }}(1)}=\frac{2}{\pi}\left[\arcsin \frac{1}{\sqrt{3}}-\sqrt{\frac{2}{3}} \operatorname{arcoth} \sqrt{3}\right] \approx 0.0495506,
\end{aligned}
$$

where $\operatorname{Vol}_{\mathrm{X}}(r)$ denotes the volume of the Bloch ball of radius $r$ in metric " $X$ ". The corresponding probability $Q(r)$ to find a qubit state $\varrho$ with positive WF within the Bloch ball of radius $r$ is depicted in Fig.1.

\subsection{Qutrit}

- The Hilbert-Schmidt metric • According to (3), the Wigner quasiprobability representation of a 3-level system is one-parametric. The spectrum of SW kernel can be parametrized by the apex angle $\zeta \in[0, \pi / 3]$ of a unit circle segment [10]:

$$
\operatorname{spec}\left(\Delta_{3}\right)=\left\{\frac{1}{3}+\frac{2}{\sqrt{3}} \sin \zeta+\frac{2}{3} \cos \zeta, \frac{1}{3}-\frac{2}{\sqrt{3}} \sin \zeta+\frac{2}{3} \cos \zeta, \frac{1}{3}-\frac{4}{3} \cos \zeta\right\} .
$$

Decomposing a qutrit density matrix spectrum via the polar coordinates $(r, \varphi)$,

$$
\operatorname{spec}(\varrho)=\left\{\frac{1}{3}-\frac{2 r}{\sqrt{3}} \cos \frac{\varphi+2 \pi}{3}, \frac{1}{3}-\frac{2 r}{\sqrt{3}} \cos \frac{\varphi+4 \pi}{3}, \frac{1}{3}-\frac{2 r}{\sqrt{3}} \cos \frac{\varphi}{3}\right\},
$$


a qutrit orbit space and its subspace of WF positivity reads $(r \geq 0, \varphi \in[0, \pi])$ :

$$
\mathcal{O}\left[\mathfrak{P}_{3}\right]: \cos \left(\frac{\varphi}{3}\right) \leq \frac{1}{2 \sqrt{3} r}, \quad \mathcal{O}\left[\mathfrak{P}_{3}^{(+)}\right]: \cos \left(\frac{\varphi}{3}+\zeta-\frac{\pi}{3}\right) \leq \frac{1}{4 \sqrt{3} r}
$$

Taking into account the expression for the Hilbert-Schmidt measure on the orbit space $\mathcal{O}\left[\mathfrak{P}_{3}\right], \omega_{\mathcal{O}\left[\mathfrak{P}_{3}\right]}=r^{7} \sin ^{2} \varphi \mathrm{d} r \wedge \mathrm{d} \varphi$, we derive the global indicator of classicality of the Hilbert-Schmidt qutrit as function of the moduli parameter $\zeta[12]$ :

$$
\mathcal{Q}_{3}(\zeta)=\frac{\int_{0}^{\pi} d \varphi \int_{0}^{\frac{1}{4 \sqrt{3} \cos \left(\frac{\varphi}{3}+\zeta-\frac{\pi}{3}\right)}} r^{7} \sin ^{2}(\varphi) d r}{\int_{0}^{\pi} d \varphi \int_{0}^{\frac{1}{2 \sqrt{3} \cos \frac{\varphi}{3}}} r^{7} \sin ^{2}(\varphi) d r}=\frac{1}{128} \frac{1+20 \cos ^{2}(\zeta-\pi / 6)}{\left(-1+4 \cos ^{2}(\zeta-\pi / 6)\right)^{5}} .
$$

Note, that the indicator $\mathcal{Q}_{3}(\zeta)$ attains at a qutrit moduli parameter $\zeta=\pi / 6$ the absolute minimum, $\min _{\zeta \in\left[0, \frac{\pi}{3}\right]} \mathcal{Q}_{3}(\zeta) \approx 0.000675$, corresponding to SW kernel with the spectrum: $\left.\operatorname{spec}\left(\Delta_{3}\right)\right|_{\zeta=\frac{\pi}{6}}=\left\|\frac{1+2 \sqrt{3}}{3}, \frac{1}{3}, \frac{1-2 \sqrt{3}}{3}\right\|$.

\section{Final remarks}

As it was outlined in the first part of our report, a true classicality/quantumness measure, being universal for different quasiprobability representations, may be sensitive to the geometry of a state space. Our calculations of the average of the global indicator $Q(\zeta)$ over a qutrit moduli space support this supposition,

$$
\left\langle Q_{\mathrm{HS}}\right\rangle_{\zeta}=0.00136368, \quad\left\langle Q_{\mathrm{B}}\right\rangle_{\zeta}=0.00019165, \quad\left\langle Q_{\mathrm{BKM}}\right\rangle_{\zeta}=0.00002762
$$

Acknowledgment The work of MB was supported in part by the EU Regional Development Fund-Project No. CZ.02.1.01/0.0/0.0/16_019/0000766.

\section{References}

[1] Weyl H., Gruppentheorie und Quantenmechanik, Hirzel-Verlag, Leipzig, 1928.

[2] Wigner E.P., On the Quantum Correction For Thermodynamic Equilibrium, Phys. Rev., V. 40, P. 749-759, 1932.

[3] Sperling J., Walmsley I.A., Quasiprobability representation of quantum coherence, Phys. Rev. A, V. 97, P. 062327-14, 2018.

[4] Veitch V., Ferrie C., Gross D., Emerson J., Negative Quasi-Probability as a Resource for Quantum Computation, New J. Phys. 2012. V. 14. P. 113011-21. 
[5] Ferrie C., Quasi-probability representations of quantum theory with applications to quantum information science, Reports on Progress in Physics, 2011. V. 74. P. 116001-24.

[6] Kenfack A., Zyczkowski K., Negativity of the Wigner function as an indicator of non-classicality, J. Opt. B: Quantum Semiclass. Opt. 2004. V. 6. P. 396-404.

[7] Spekkens R. W., Negativity and contextuality are equivalent notions of nonclassicality, Phys. Rev. Lett. 2008. V. 101. P. 020401-4.

[8] Hudson R.L., When is the Wigner quasi-probability density non-negative?, Rep. Math. Phys. 1974. V. 6. P. 240-252.

[9] Stratonovich R.L., On Distributions in Representation Space, Soviet Physics JETP. 1957. V. 4. P. 891-898. (Russian original - ZhETF. 1957. V. 31, No. 6, P. 1012.)

[10] Abgaryan V., Khvedelidze A., On families of Wigner functions for $N$-level quantum systems, Preprint https://arxiv.org/pdf/1708.05981.pdf. 2018. 8 p.

[11] Abgaryan A., Khvedelidze A., Torosyan A., On the moduli space of the Wigner quasiprobability distributions for $N$-dimensional quantum systems, J. Math. Sci. 2018. V. 240. P. 617-633.

[12] Abgaryan V., Khvedelidze A., Torosyan A., The global indicator of classicality of an arbitrary $N$-level quantum system, Zap. Nauch. Sem. POMI. 2019. V. 485. P. $5-23$.

[13] Morozova E.A., Chentsov N.N., Markov invariant geometry on state manifolds, J. Math. Sci. 1991. V. 56. P. 2648-2669 (Translated from: Itogi Nauki i Tekhniki. 1990. V. 36. P. 69-102.)

[14] Petz D., Sudar C., Geometries of quantum states, J. Math. Phys. 1996. V. 37. P. 2662-2673. 